\title{
The Effects of Assisted Ergometer Training With a Functional Electrical Stimulation on Exercise Capacity and Functional Ability in Subacute Stroke Patients
}

\author{
So Young Lee, $\mathrm{MD}^{1}$, Sa-Yoon Kang, $\mathrm{MD}^{2}$, Sang Hee Im, MD, PhD ${ }^{3}$, Bo Ryun Kim, $\mathrm{MD}^{1}$, \\ Sun Mi Kim, MD ${ }^{1}$, Ho Min Yoon, $\mathrm{MS}^{1}$, Eun Young Han, $\mathrm{MD}^{1}$ \\ Departments of ${ }^{1}$ Rehabilitation Medicine and ${ }^{2}$ Neurology, Jeju National University Hospital, \\ Jeju National University College of Medicine, Jeju; \\ ${ }^{3}$ Department of Rehabilitation Medicine, Kwandong University College of Medicine, Gangneung, Korea
}

Objective To determine if assistive ergometer training can improve the functional ability and aerobic capacity of subacute stroke patients and if functional electrical stimulation (FES) of the paretic leg during ergometer cycling has additional effects.

Methods Sixteen subacute stroke patents were randomly assigned to the FES group $(n=8)$ or the control group $(\mathrm{n}=8)$. All patients underwent assistive ergometer training for 30 minutes (five times per week for 4 weeks). The electrical stimulation group received FES of the paretic lower limb muscles during assistive ergometer training. The six-minute walk test (6MWT), Berg Balance Scale (BBS), and the Korean version of Modified Barthel Index (K-MBI) were evaluated at the beginning and end of treatment. Peak oxygen consumption $\left(\mathrm{Vo}_{2 \text { peak }}\right)$, metabolic equivalent (MET), resting and maximal heart rate, resting and maximal blood pressure, maximal rate pressure product, submaximal rate pressure product, submaximal rate of perceived exertion, exercise duration, respiratory exchange ratio, and estimated anaerobic threshold (AT) were determined with the exercise tolerance test before and after treatment.

Results At 4 weeks after treatment, the FES assistive ergometer training group showed significant improvements in 6 MWT $(p=0.01)$, BBS $(p=0.01)$, K-MBI $(p=0.01), V_{2 p e a k}(p=0.02)$, MET $(p=0.02)$, and estimated AT $(p=0.02)$. The control group showed improvements in only BBS $(\mathrm{p}=0.01)$ and $\mathrm{K}-\mathrm{MBI}(\mathrm{p}=0.02)$. However, there was no significant difference in exercise capacity and functional ability between the two groups.

Conclusion This study demonstrated that ergometer training for 4 weeks improved the functional ability of subacute stroke patients. In addition, aerobic capacity was improved after assisted ergometer training with a FES only.

Keywords Electrical stimulation, Ergometry, Bicycling, Stroke, Oxygen consumption 


\section{INTRODUCTION}

Stroke is a leading cause of disability that impairs ambulatory activity and daily living functions $[1,2]$. Poststroke functional deficits, such as postural imbalance, muscle weakness, or spasticity may promote physical inactivity and a deconditioning state, which can lead to reduced cardiovascular fitness [2-6]. The low aerobic capacity eventually results in low ambulatory function, social participation, and quality of life [4]. Therefore, it is essential to encourage aerobic exercise in post-stroke patients, but very few can participate in intensive gait training immediately after their stroke event [7], because of muscle weakness, fatigue, or general deconditioning [8].

Cycling exercise is a safe, functional treatment that is accessible by these patients for the relearning of motor skills required for locomotion $[9,10]$. Pedaling is an aerobic exercise and a repetitive, functional activity, which requires reciprocal contraction of agonist and antagonist muscles of the lower limbs in a repeating pattern similar to that required for walking [11]. The motorized cycling ergometer is an especially useful treatment for severe disabled stroke patients, as automatic pedaling facilitates phasic, coordinated muscle activity with continuous use of the hemiside extremity [12]. This exercise improves cardiovascular fitness, such as peak oxygen consumption, workload, and endurance [13]. Also, because cycling is practiced in the sitting position, it is safe, easy to perform, and can be widely applied, regardless of the severity of motor impairment [14]. Therefore, cycling has been proposed to have potential patient benefit when used as an adjunct to therapy after stroke [13,15-19].

In addition, functional electrical stimulation (FES) exercise can increase strength and prevent atrophy of paralyzed muscles by using stimulation patterns that induce repetitive contractions in selected muscle groups [20]. In a previous study [21], we reported improvement of cardiovascular function after the application of electrical stimulation in healthy adults. FES could be an alternative strategy to increase the muscle work performed in severely disabled patients [22,23].

Motorized cycle ergometers combined with FES became available in 1984 and are used primarily as a therapy for people with spinal cord injury (SCI) [24]. Several studies have reported using the FES-evoked leg cycle ergometer as a rehabilitation therapy for lower limb paralysis following SCI [25-31]. The therapy was found to increase the number of activated muscle fibers, muscle mass, strength, functional performance, and circulation.

However, no previous study has investigated the effectiveness of assisted ergometer training with a FES on hemiparetic legs in subacute stroke patients, including evaluation of the patient's functional ability and aerobic capacity. Therefore, the aim of this study was to evaluate assisted ergometer training with a FES in subacute stroke patients for improving their functional ability and aerobic capacity. We also aimed to determine if concomitant FES provides benefits in addition to the ergometer alone.

\section{MATERIALS AND METHODS}

\section{Subjects}

This study was conducted in 16 stroke patients who had been treated in the rehabilitation department of our hospital. All subjects provided written informed consent before the selection procedure. The study protocol was reviewed and approved by the Institutional Review Board. The participants 1) suffered a primary ischemic or hemorrhagic stroke as revealed by computed tomography or magnetic resonance imaging scans, 2) had an onset of stroke within 6 months, and 3) presented with mild to moderate paresis of the lower extremities (manual muscle function test $>$ grade 2). Patients who had 1) severe deficits in communication, memory, or understanding, 2) any additional neurological or orthopedic disease causing motor deficits, such as fractures, joint degenerative changes, or clinical instability of the hip or knee joint, 3) psychiatric problems or unstable cardiorespiratory disease, or 4) inability to tolerate, or contraindications to electrical stimulation-e.g., epilepsy, cancer, skin ulcer, pacemaker, pregnancy-were excluded.

\section{Methods}

Eligible patients were randomly assigned to two groups. Eight patients (mean age, $63.25 \pm 15.00$ years) were assigned to the electrical stimulation group and another eight patients (mean age, $63.25 \pm 14.12$ years) to the control group. All patients underwent assistive ergometer training five times per week for 4 weeks to maintain a pedaling cadence of $30 \mathrm{rpm}$ for 30 minutes. The electrical stimulation group received FES on the paretic quadriceps, hamstring, gluteus maximus, and tibialis anterior muscles using two EMG-FES 2000 (Cyber Medic, Seoul, Korea) during assistive ergometer training. One was ap- 
plied to paretic quadriceps and tibialis anterior, and the other was applied to hamstring and gluteus maximus. The stimulation protocol consisted of a symmetrical biphasic square pulse at $60 \mathrm{~Hz}$, a constant pulse width of $300 \mu \mathrm{s}$, and a duty cycle of 1.0 second on and 1.0 second off alternatively. We endeavored to synchronize FES stimulation timing to the cycling movement according to each muscle activation pattern, although we could not use an automatic synchronizing FES ergometer. The maximum power output of the stimulator was $100 \mathrm{~mA}$. The stimulation intensity was increased within the range of motor threshold to the maximal comfortably tolerated intensity. Both groups underwent the symptom-limited low velocity graded treadmill test using a modified Harbor protocol $[32,33]$. The exercise test was performed using a CH2000 (Cambridge Heart Inc., Tewksbury, MA, USA) integrated 12-lead ECG for stress testing, Quark CPET (Cosmed, Rome, Italy) breath by breath gas exchange data analysis, a T-2100 treadmill (GE Healthcare, Fairfield, CT, USA), and a blood pressure and heart rate monitor. The initial round of treadmill walking without an incline was used to assess gait safety and to select the target walking velocity for subsequent maximal-effort graded treadmill testing. The zero-incline treadmill test was started at $0.5 \mathrm{mph}$ and slowly advanced by $0.1 \mathrm{mph}$ increments according to the patient's subjective tolerance and observer-rated gait stability. Patients capable of performing $\geq 3$ consecutive minutes of treadmill walking at $\geq 0.5 \mathrm{mph}$ were allowed 15 minutes of seated rest, after which they underwent constant-velocity, progressively graded treadmill exercise testing. For the initial 2 minutes, patients walked on the treadmill without an incline, followed by 2 minutes at $4 \%$ incline, with a $2 \%$ increase per 2 minutes thereafter. The exercise test was terminated on patient request or in the presence of gait instability or cardiovascular decompensation sign. Handrail support was minimally allowed, and a gait belt support and close supervision were provided as safety measures.

Peak oxygen consumption $\left(\mathrm{Vo}_{2 \text { peak }}\right)$, metabolic equivalent (MET), resting and maximal heart rate (RHR, MHR), resting and maximal blood pressure (RBP, MBP), maximal rate pressure product (MRPP), exercise duration, respiratory exchange ratio (RER), estimated anaerobic threshold (AT), submaximal rate pressure product (SRPP), and submaximal rate of perceived exertion (SRPE) - Borg Scale, from 6 to 20-were determined before and after the exercise test. In addition, functional exercise capacity measured by the six-minute walk test (6MWT) in a $50 \mathrm{~m}$ in-hospital corridor, Berg Balance Scale (BBS), and the Korean version of the Modified Barthel Index (K-MBI) were evaluated at the beginning and the end of treatment.

\section{Statistical analysis}

Statistical analysis was performed using SPSS ver. 18.0 (SPSS Inc., Chicago, IL, USA) for Windows. The chisquare and Mann-Whitney tests were used to compare the baseline characteristics of the two groups. Wilcoxon rank sum test was also used to compare the exercise capacity and functional ability within each group at baseline and 4 weeks after testing. Repeated measures analysis of variance was used for the inter-group comparison of all parameters of exercise capacity and functional ability between the two groups according to the time period until 4 weeks. The null hypothesis of no difference was rejected if $\mathrm{p}$-values were less than 0.05 .

\section{RESULTS}

\section{General characteristics of the participants}

The baseline characteristics of the participants are summarized in Tables 1 and 2. Eight participants were assigned to each study group and each included four men. The mean age at enrolment was $63.25 \pm 15.00$ years in the electrical stimulation group and $63.25 \pm 14.12$ years in the control group. Demographic characteristics including gender, age, time since cardiovascular accident, height, weight, and baseline cardiopulmonary functions of the two groups were not significantly different.

Changes of parameters before and after treatment within each group

At 4 weeks after treatment, the FES assistive ergometer training group showed a significant improvement in both exercise capacity and functional ability (Table 3 ). The 6 MWT increased from $193.63 \pm 102.97$ to $266.25 \pm 113.38$ $(\mathrm{p}=0.01)$, BBS increased from $43.88 \pm 7.14$ to $47.13 \pm 6.98$ $(\mathrm{p}=0.01)$, and K-MBI increased from $76.38 \pm 10.64$ to $84.00 \pm 8.59(\mathrm{p}=0.01)$. In addition, $\mathrm{Vo}_{2 \text { peak }}$ increased from $16.00 \pm 2.79$ to $17.83 \pm 3.09$ ( $\mathrm{p}=0.02)$, MET increased from $4.55 \pm 0.82$ to $17.83 \pm 3.09(\mathrm{p}=0.02)$, and the estimated AT increased from $11.35 \pm 1.36$ to $13.72 \pm 2.49(\mathrm{p}=0.02)$. The control group showed no significant changes in exercise capacity, whereas statistically significant improvements 
Table 1. Baseline characteristics of the two groups

\begin{tabular}{lccc}
\hline \multicolumn{1}{c}{ Characteristic } & FES+ergometer $(\mathbf{n = 8})$ & Ergometer $(\mathbf{n}=\mathbf{8})$ & p-value \\
\hline Gender (male:female) & $4: 4$ & $4: 4$ & \\
Age (yr) & $63.25 \pm 15.00$ & $63.25 \pm 14.12$ & 0.96 \\
Infarction:hemorrhage & $4: 4$ & $6: 2$ & \\
Side of lesion (right:left) & $5: 3$ & $5: 3$ & 0.88 \\
Time since CVA (day) & $62.50 \pm 52.23$ & $57.38 \pm 34.63$ & 0.38 \\
Height (cm) & $158.13 \pm 8.80$ & $162.13 \pm 9.37$ & 0.96 \\
\hline Weight (kg) & $60.76 \pm 7.26$ & $55.00 \pm 22.96$ & \\
\hline
\end{tabular}

Values are presented as mean \pm standard deviation or number.

FES, functional electrical stimulation; CVA, cerebrovascular accident.

$\mathrm{p}<0.05$.

Table 2. Exercise capacity and functional ability at baseline

\begin{tabular}{lccc}
\hline & FES+ergometer & Ergometer & p-value \\
\hline 6 MWT & $193.63 \pm 102.97$ & $291.00 \pm 82.61$ & 0.07 \\
BBS & $43.88 \pm 7.14$ & $47.38 \pm 5.66$ & 0.20 \\
\hline K-MBI & $76.38 \pm 10.64$ & $83.38 \pm 10.23$ & 0.28 \\
\hline MI & $70.00 \pm 4.54$ & $72.50 \pm 6.02$ & 0.51 \\
\hline Vo $_{\text {2peak }}$ & $16.00 \pm 2.79$ & $20.08 \pm 5.79$ & 0.16 \\
MET & $4.55 \pm 0.82$ & $5.75 \pm 1.66$ & 0.13 \\
\hline RHR & $87.50 \pm 12.72$ & $94.50 \pm 21.80$ & 0.72 \\
\hline MHR & $128.50 \pm 25.82$ & $143.38 \pm 28.08$ & 0.28 \\
\hline RsBP & $127.88 \pm 18.95$ & $123.63 \pm 19.60$ & 0.72 \\
RdBP & $77.63 \pm 13.24$ & $68.17 \pm 7.20$ & 0.11 \\
\hline MsBP & $177.75 \pm 18.24$ & $173.13 \pm 34.01$ & 0.80 \\
\hline MdBP & $79.63 \pm 16.62$ & $80.75 \pm 19.52$ & 0.96 \\
\hline MRPP & $23.017 .13 \pm 6,007.24$ & $24,898.63 \pm 7,445.89$ & 0.80 \\
AT & $11.35 \pm 1.36$ & $13.61 \pm 4.40$ & 0.38 \\
\hline SRPP & $1703.25 \pm 4,164.66$ & $16,747.50 \pm 5,174.25$ & 0.96 \\
\hline SRPE & $12.00 \pm 1.52$ & $11.00 \pm 1.52$ & 0.33 \\
\hline RER & $0.97 \pm 0.15$ & $0.98 \pm 0.12$ & 0.80 \\
\hline Ex Dur & $506.88 \pm 193.13$ & $697.50 \pm 285.73$ & 0.13 \\
\hline
\end{tabular}

Values are presented as mean \pm standard deviation.

FES, functional electrical stimulation; 6MWT, 6 minute walk test; BBS, Berg Balance Scale; K-MBI, Korean-Modified Barthel Index; MI, Motricity Index; $\mathrm{Vo}_{2 \text { peak }}$ peak oxygen consumption; MET, metabolic equivalent; RHR, resting heart rate; MHR, maximal heart rate; RsBP, resting systolic blood pressure; RdBP, resting diastolic blood pressure; MsBP, maximal systolic blood pressure; MdBP, maximal diastolic blood pressure; MRPP, maximal rate pressure product; AT, estimated anaerobic threshold; SRPP, submaximal rate pressure product; SRPE, submaximal rate of perceived exertion; RER, respiratory exchange ratio; Ex Dur, exercise duration. $\mathrm{p}<0.05$.

occurred in the BBS $(\mathrm{p}=0.01)$ and the K-MBI $(\mathrm{p}=0.02)$.

Changes of parameters before and after treatment between the two groups

After 4 weeks, no significant intergroup differences were found in the exercise capacity and functional ability 
Table 3. Study parameters measured before and after treatment within each study group

\begin{tabular}{|c|c|c|c|c|c|c|}
\hline \multirow{2}{*}{ Parameter } & \multicolumn{3}{|c|}{ FES+ergometer } & \multicolumn{3}{|c|}{ Ergometer } \\
\hline & Before & After & p-value & Before & After & p-value \\
\hline 6MWT & $193.63 \pm 102.97$ & $266.25 \pm 113.38$ & $0.01^{\text {a) }}$ & $291.00 \pm 82.61$ & $331.50 \pm 60.22$ & 0.23 \\
\hline BBS & $43.88 \pm 7.14$ & $47.13 \pm 6.98$ & $0.01^{\mathrm{a})}$ & $47.38 \pm 5.66$ & $51.63 \pm 2.50$ & $0.01^{\text {a) }}$ \\
\hline K-MBI & $76.38 \pm 10.64$ & $84.00 \pm 8.59$ & $0.01^{\text {a) }}$ & $83.38 \pm 10.23$ & $90.25 \pm 8.40$ & $0.02^{\mathrm{a})}$ \\
\hline $\mathrm{Vo}_{\text {2peak }}$ & $16.00 \pm 2.79$ & $17.83 \pm 3.09$ & $0.02^{\text {a) }}$ & $20.08 \pm 5.79$ & $20.30 \pm 7.00$ & 1.00 \\
\hline MET & $4.55 \pm 0.82$ & $5.10 \pm 0.87$ & $0.02^{\mathrm{a})}$ & $5.75 \pm 1.66$ & $5.79 \pm 2.01$ & 1.00 \\
\hline RHR & $87.50 \pm 12.72$ & $91.13 \pm 11.97$ & 0.67 & $94.50 \pm 21.80$ & $91.00 \pm 20.63$ & 0.73 \\
\hline MHR & $128.50 \pm 25.82$ & $123.38 \pm 23.93$ & 0.29 & $143.38 \pm 28.08$ & $135.50 \pm 25.54$ & 0.44 \\
\hline RsBP & $127.88 \pm 18.95$ & $133.63 \pm 17.94$ & 0.23 & $123.63 \pm 19.60$ & $124.13 \pm 21.10$ & 0.89 \\
\hline RdBP & $77.63 \pm 13.24$ & $73.13 \pm 12.68$ & 0.07 & $68.17 \pm 7.20$ & $72.17 \pm 12.67$ & 0.35 \\
\hline MsBP & $177.75 \pm 18.24$ & $186.63 \pm 24.21$ & 0.14 & $173.13 \pm 34.01$ & $177.00 \pm 22.25$ & 0.67 \\
\hline MdBP & $79.63 \pm 16.62$ & $84.13 \pm 19.80$ & 0.24 & $80.75 \pm 19.52$ & $76.25 \pm 13.93$ & 0.36 \\
\hline MRPP & $23,017.13 \pm 6,007.24$ & $23,385.75 \pm 5,728.40$ & 0.87 & $24,898.63 \pm 7,445.89$ & $24,141.50 \pm 5,865.65$ & 0.74 \\
\hline $\mathrm{AT}$ & $11.35 \pm 1.36$ & $13.72 \pm 2.49$ & $0.02^{\text {a) }}$ & $13.61 \pm 4.40$ & $14.71 \pm 4.42$ & 0.07 \\
\hline RER & $0.97 \pm 0.15$ & $0.98 \pm 0.18$ & 0.34 & $0.98 \pm 0.12$ & $0.99 \pm 0.12$ & 0.67 \\
\hline SRPP & $17,303.25 \pm 4,164.66$ & $17,035.75 \pm 4,213.58$ & 0.58 & $16,747.50 \pm 5,174.25$ & $16,441.88 \pm 5,092.21$ & 1.00 \\
\hline SRPE & $12.00 \pm 1.52$ & $11.75 \pm 1.04$ & 0.66 & $11.00 \pm 1.52$ & $12.25 \pm 1.49$ & 0.20 \\
\hline Ex Dur & $506.88 \pm 193.13$ & $562.50 \pm 174.38$ & 0.67 & $697.50 \pm 285.73$ & $735.00 \pm 264.83$ & 0.48 \\
\hline
\end{tabular}

Values are presented as mean \pm standard deviation.

FES, functional electrical stimulation; 6MWT, 6 minute walk test; BBS, Berg Balance Scale; K-MBI, Korean-Modified Barthel Index; MI, Motricity Index; $\mathrm{Vo}_{2 \text { peak }}$, peak oxygen consumption; MET, metabolic equivalent; RHR, resting heart rate; MHR, maximal heart rate; RsBP, resting systolic blood pressure; RdBP, resting diastolic blood pressure; MsBP, maximal systolic blood pressure; MdBP, maximal diastolic blood pressure; MRPP, maximal rate pressure product; AT, estimated anaerobic threshold; SRPP, submaximal rate pressure product; SRPE, submaximal rate of perceived exertion; RER, respiratory exchange ratio; Ex Dur, exercise duration.

${ }^{\mathrm{a})} \mathrm{p}<0.05$.

(Table 4).

\section{DISCUSSION}

The results of this study provide evidence that FES assistive ergometer training in the hemiparetic muscles of subacute stroke patients improved their aerobic capacities as well as functional abilities.

Several investigations $[5,8,28,30,34-38]$ have demonstrated similar beneficial effects of FES assistive ergometer training in patients with SCI, heart failure, and stroke. Szecsi et al. [5] assessed whether FES-assisted cycling on the paretic leg in subjects with subacute stroke hemiparesis could improve quantifiable biomechanical parameters such as smoothness, power, and symmetry of cycling. They showed that FES-assisted cycling was associated with strengthening the affected leg, reestablishing the side balance of forces, and improving pedaling control, particularly in patients with hemiparesis in the early phase of convalescence after stroke. Although they did not evaluate functional abilities and aerobic capacities, they noted that objective goals, such as functional outcomes can be achieved with the clinical application of this therapy. Lo et al. [37] also reported that cycling exercise with FES on the hemiparetic leg improved postural control and spasticity of the extremities in subacute stroke patients. They suggested that the application of FES to the affected leg during cycling training has additional benefits, especially for patients with high muscle tone. These findings emphasized the beneficial effects of FES on hemiparetic legs specifically in subacute stroke patients.

Additionally, FES-induced cycling on the both legs (hemiside and intact-side) has demonstrated significant improvement in lower extremity strength and functional ability in subacute stroke patients. Ferrante et al. [35] and 
Table 4. Inter-group comparisons of parameters according to time by repeated-measures analysis of variance

\begin{tabular}{|c|c|c|c|c|c|}
\hline \multirow{2}{*}{ Parameter } & \multicolumn{2}{|c|}{ FES+ergometer } & \multicolumn{2}{|c|}{ Ergometer } & \multirow{2}{*}{ p-value } \\
\hline & Before & After & Before & After & \\
\hline $6 \mathrm{MWT}$ & $193.63 \pm 102.97$ & $266.25 \pm 113.38$ & $291.00 \pm 82.61$ & $331.50 \pm 60.22$ & 0.42 \\
\hline BBS & $43.88 \pm 7.14$ & $47.13 \pm 6.98$ & $47.38 \pm 5.66$ & $51.63 \pm 2.50$ & 0.56 \\
\hline K-MBI & $76.38 \pm 10.64$ & $84.00 \pm 8.59$ & $83.38 \pm 10.23$ & $90.25 \pm 8.40$ & 0.81 \\
\hline $\mathrm{Vo}_{\text {2peak }}$ & $16.00 \pm 2.79$ & $17.83 \pm 3.09$ & $20.08 \pm 5.79$ & $20.30 \pm 7.00$ & 0.36 \\
\hline MET & $4.55 \pm 0.82$ & $5.10 \pm 0.87$ & $5.75 \pm 1.66$ & $5.79 \pm 2.01$ & 0.31 \\
\hline RHR & $87.50 \pm 12.72$ & $91.13 \pm 11.97$ & $94.50 \pm 21.80$ & $91.00 \pm 20.63$ & 0.53 \\
\hline MHR & $128.50 \pm 25.82$ & $123.38 \pm 23.93$ & $143.38 \pm 28.08$ & $135.50 \pm 25.54$ & 0.78 \\
\hline RsBP & $127.88 \pm 18.95$ & $133.63 \pm 17.94$ & $123.63 \pm 19.60$ & $124.13 \pm 21.10$ & 0.47 \\
\hline $\mathrm{RdBP}$ & $77.63 \pm 13.24$ & $73.13 \pm 12.68$ & $68.17 \pm 7.20$ & $72.17 \pm 12.67$ & 0.06 \\
\hline MsBP & $177.75 \pm 18.24$ & $186.63 \pm 24.21$ & $173.13 \pm 34.01$ & $177.00 \pm 22.25$ & 0.73 \\
\hline MdBP & $79.63 \pm 16.62$ & $84.13 \pm 19.80$ & $80.75 \pm 19.52$ & $76.25 \pm 13.93$ & 0.13 \\
\hline MRPP & $23,017.13 \pm 6,007.24$ & $23,385.75 \pm 5,728.40$ & $24,898.63 \pm 7,445.89$ & $24,141.50 \pm 5,865.65$ & 0.76 \\
\hline AT & $11.35 \pm 1.36$ & $13.72 \pm 2.49$ & $13.61 \pm 4.40$ & $14.71 \pm 4.42$ & 0.26 \\
\hline RER & $0.97 \pm 0.15$ & $0.98 \pm 0.18$ & $0.98 \pm 0.12$ & $0.99 \pm 0.12$ & 1.00 \\
\hline SRPP & $17,303.25 \pm 4,164.66$ & $17,035.75 \pm 4,213.58$ & $16,747.50 \pm 5,174.25$ & $16,441.88 \pm 5,092.21$ & 0.99 \\
\hline SRPE & $12.00 \pm 1.52$ & $11.75 \pm 1.04$ & $11.00 \pm 1.52$ & $12.25 \pm 1.49$ & 0.19 \\
\hline Ex Dur & $506.88 \pm 193.13$ & $562.50 \pm 174.38$ & $697.50 \pm 285.73$ & $735.00 \pm 264.83$ & 0.84 \\
\hline
\end{tabular}

Values are presented as mean \pm standard deviation.

FES, functional electrical stimulation; 6MWT, 6 minute walk test; BBS, Berg Balance Scale; K-MBI, Korean-Modified Barthel Index; $\mathrm{Vo}_{2 \text { peak }}$, peak oxygen consumption; MET, metabolic equivalent; RHR, resting heart rate; MHR, maximal heart rate; RsBP, resting systolic blood pressure; RdBP, resting diastolic blood pressure; MsBP, maximal systolic blood pressure; MdBP, maximal diastolic blood pressure; MRPP, maximal rate pressure product; AT, estimated anaerobic threshold; SRPP, submaximal rate pressure product; SRPE, submaximal rate of perceived exertion; RER, respiratory exchange ratio; Ex Dur, exercise duration.

$\mathrm{p}<0.05$.

Ambrosini et al. [36] evaluated the effectiveness of FESinduced cycling on both legs in subacute stroke patients. Their studies revealed that FES cycling training significantly improved lower extremity strength, motor control, motor recovery, and walking abilities, such as Motricity Index, Trunk Control Test, Upright Motor Control Test, and maximum isometric voluntary contraction compared to cycling only. These results confirmed that FES cycling training applied in the subacute stroke phase may play an important role in facilitating and accelerating motor recovery. The investigators concluded that the improvements they observed resulted from increased sensorial input provided to the brain by FES.

The improvements observed through the 6MWT, BBS, and K-MBI in our study are in line with the results cited above. However, in our study, FES applied only to the muscles on the hemiparetic side was effective in improving functional abilities. This suggests that FES-induced cycling may facilitate coordinated muscle activity, relearning of how to execute movements of paretic muscles voluntarily, and induction of continuous use of the hemiside extremity.

Janssen et al. [8] reported that their FES assistive cycling training program on paretic lower extremities in chronic stroke patients improved cycling performance, functional performance, and aerobic exercise, such as power of output (POmax), $\mathrm{Vo}_{2 \text { peak }}, 6 \mathrm{MWT}$, and BBS performance. Except for muscle strength, it had no additional effect over assistive ergometer only. Our study reached a similar conclusion in subacute stroke patients in that it showed improvements in aerobic capacities, such as $\mathrm{Vo}_{2 \text { peak }}$, MET, and estimated AT in the FES assistive ergometer training group within 4 weeks. These results could be explained by repetitive voluntary contraction of leg muscles as an adaptation to FES, which also promotes circulatory adaptations, hypertrophy, and histochemical changes in mus- 
cles, all of which are helpful in relearning how to execute movements. The changes in response to therapy increase metabolic capacity, prevent muscle atrophy, and induce functional recovery. Also, FES helps patients to focus on treatment during cycling, thus improving the efficiency of the exercise [36]. Moreover, improvement in estimated AT provides meaningful evidence that FES could be used to improve exercise endurance. Also, our study found that FES plus assistive motorized cycling was beneficial for aerobic capacity in subacute as well as chronic stroke patients.

There are several study limitations. The most serious limitation is the small number of subjects evaluated, making it insufficient to monitor the effects of FES training. Second, a therapeutic intervention period of 4 weeks might be too short to demonstrate the added effects of FES training, compared to the control. Third, we could not evaluate the changes in muscle strength, mass, and muscle spasticity, although they might have influenced the functional ability. Fourth, we could not perfectly synchronize FES stimulation timing with the angular movement of the ergometer motor. Finally, we did not use the cycling graded exercise stress test, which could be applied in the sitting position. Thus, symptom-limited exercise tests had to be performed by walking or running, and the participants might have already reached functional recovery at baseline exercise test. Our results support previous additional, large-scale, long-term followup studies, including FES synchronization and other relevant factors, evaluating functional ability and aerobic capacity after FES-assisted ergometer training.

In conclusion, this study demonstrated that ergometer training for 4 weeks improved the functional ability of subacute stroke patients. In addition, aerobic capacity was improved after assisted ergometer training with a FES only. Therefore, we suggest assisted ergometer training with a FES as a useful alternative therapy for increasing the aerobic capacity of subacute stroke patients.

\section{CONFLICT OF INTEREST}

No potential conflict of interest relevant to this article was reported.

\section{ACKNOWLEDGMENTS}

This work was supported by a research grant from the
Medical Research Fund (institute of medical science academic research fund) of Jeju National University in 2011.

\section{REFERENCES}

1. Bobath B. Adult hemiplegia: evaluation and treatment. 3rd ed. London: Butterworth-Heinemann; 1990.

2. Gresham GE, Fitzpatrick TE, Wolf PA, McNamara PM, Kannel WB, Dawber TR. Residual disability in survivors of stroke: the Framingham study. N Engl J Med 1975;293:954-6.

3. Brown DA, Kautz SA. Increased workload enhances force output during pedaling exercise in persons with poststroke hemiplegia. Stroke 1998;29:598-606.

4. Mayo NE, Wood-Dauphinee S, Ahmed S, Gordon C, Higgins J, McEwen S, et al. Disablement following stroke. Disabil Rehabil 1999;21:258-68.

5. Szecsi J, Krewer C, Muller F, Straube A. Functional electrical stimulation assisted cycling of patients with subacute stroke: kinetic and kinematic analysis. Clin Biomech (Bristol, Avon) 2008;23:1086-94.

6. Jorgensen HS, Nakayama H, Raaschou HO, Olsen TS. Stroke: neurologic and functional recovery the Copenhagen Stroke Study. Phys Med Rehabil Clin N Am 1999;10:887-906.

7. Hwang IS, Lee HM, Cherng RJ, Chen JJ. Electromyographic analysis of locomotion for healthy and hemiparetic subjects: study of performance variability and rail effect on treadmill. Gait Posture 2003;18:1-12.

8. Janssen TW, Beltman JM, Elich P, Koppe PA, Konijnenbelt $\mathrm{H}$, de Haan A, et al. Effects of electric stimulation-assisted cycling training in people with chronic stroke. Arch Phys Med Rehabil 2008;89:463-9.

9. Hunt KJ, Stone B, Negard NO, Schauer T, Fraser MH, Cathcart AJ, et al. Control strategies for integration of electric motor assist and functional electrical stimulation in paraplegic cycling: utility for exercise testing and mobile cycling. IEEE Trans Neural Syst Rehabil Eng 2004;12:89-101.

10. Takahashi T, Takazawa M, Nishiyama Y, Nakano E, Handa Y. FES cycling chair for the lower limbs disabled people with electric motor power assist. In: Proceedings of the 9th Annual International FES Society Conference; 2004 Sep 6-9; Bournemouth, UK. p. 2479.

11. Raasch CC, Zajac FE. Locomotor strategy for pedaling: 
muscle groups and biomechanical functions. J Neurophysiol 1999;82:515-25.

12. Hancock NJ, Shepstone L, Winterbotham W, Pomeroy $\mathrm{V}$. Effects of lower limb reciprocal pedalling exercise on motor function after stroke: a systematic review of randomized and nonrandomized studies. Int J Stroke 2012;7:47-60.

13. Potempa K, Lopez M, Braun LT, Szidon JP, Fogg L, Tincknell T. Physiological outcomes of aerobic exercise training in hemiparetic stroke patients. Stroke 1995;26:101-5.

14. Brown DA, Kautz SA, Dairaghi CA. Muscle activity adapts to anti-gravity posture during pedalling in persons with post-stroke hemiplegia. Brain 1997;120(Pt 5):825-37.

15. Holt R, Kendrick C, McGlashan K, Kirker S, Jenner J. Static bicycle training for functional mobility in chronic stroke: case report. Physiotherapy 2001;87:257-60.

16. Fujiwara T, Liu M, Tanuma A, Hase K, Tsuji T. Pedaling exercise for neuromuscular re-education: a review. Crit Rev Phys Rehabil Med 2005;17:163-78.

17. Katz-Leurer M, Sender I, Keren O, Dvir Z. The influence of early cycling training on balance in stroke patients at the subacute stage: results of a preliminary trial. Clin Rehabil 2006;20:398-405.

18. Quaney BM, Boyd LA, McDowd JM, Zahner LH, He J, Mayo MS, et al. Aerobic exercise improves cognition and motor function poststroke. Neurorehabil Neural Repair 2009;23:879-85.

19. Katz-Leurer M, Carmeli E, Shochina M. The effect of early aerobic training on independence six months post stroke. Clin Rehabil 2003;17:735-41.

20. Sheffler LR, Chae J. Neuromuscular electrical stimulation in neurorehabilitation. Muscle Nerve 2007;35:562-90.

21. Lee SY, Im SH, Kim BR, Choi JH, Lee SJ, Han EY. The effects of neuromuscular electrical stimulation on cardiopulmonary function in healthy adults. Ann Rehabil Med 2012;36:849-56.

22. Kesar TM, Perumal R, Jancosko A, Reisman DS, Rudolph KS, Higginson JS, et al. Novel patterns of functional electrical stimulation have an immediate effect on dorsiflexor muscle function during gait for people poststroke. Phys Ther 2010;90:55-66.

23. Santos M, Zahner LH, McKiernan BJ, Mahnken JD, Quaney B. Neuromuscular electrical stimulation improves severe hand dysfunction for individuals with chronic stroke: a pilot study. J Neurol Phys Ther 2006;30:175-83.

24. Petrofsky JS, Phillips CA. The use of functional electrical stimulation for rehabilitation of spinal cord injured patients. Cent Nerv Syst Trauma 1984;1:57-74.

25. Petrofsky JS, Laymon M. The effect of previous weight training and concurrent weight training on endurance for functional electrical stimulation cycle ergometry. Eur J Appl Physiol 2004;91:392-8.

26. Petrofsky JS, Stacy R. The effect of training on endurance and the cardiovascular responses of individuals with paraplegia during dynamic exercise induced by functional electrical stimulation. Eur J Appl Physiol Occup Physiol 1992;64:487-92.

27. Hooker SP, Scremin AM, Mutton DL, Kunkel CF, Cagle G. Peak and submaximal physiologic responses following electrical stimulation leg cycle ergometer training. J Rehabil Res Dev 1995;32:361-6.

28. Skold C, Lonn L, Harms-Ringdahl K, Hultling C, Levi $\mathrm{R}$, Nash M, et al. Effects of functional electrical stimulation training for six months on body composition and spasticity in motor complete tetraplegic spinal cord-injured individuals. J Rehabil Med 2002;34:2532.

29. Nash MS, Montalvo BM, Applegate B. Lower extremity blood flow and responses to occlusion ischemia differ in exercise-trained and sedentary tetraplegic persons. Arch Phys Med Rehabil 1996;77:1260-5.

30. Raymond J, Schoneveld K, Van Kemenade CH, Davis GM. Onset of electrical stimulation leg cycling in individuals with paraplegia. Med Sci Sports Exerc 2002;34:1557-62.

31. Raymond J, Davis GM, Climstein M, Sutton JR. Cardiorespiratory responses to arm cranking and electrical stimulation leg cycling in people with paraplegia. Med Sci Sports Exerc 1999;31:822-8.

32. Buchfuhrer MJ, Hansen JE, Robinson TE, Sue DY, Wasserman K, Whipp BJ. Optimizing the exercise protocol for cardiopulmonary assessment. J Appl Physiol 1983;55:1558-64.

33. Macko RF, Katzel LI, Yataco A, Tretter LD, DeSouza CA, Dengel DR, et al. Low-velocity graded treadmill stress testing in hemiparetic stroke patients. Stroke 1997;28:988-92.

34. Ferrante S, Pedrocchi A, Ferrigno G, Molteni F. FES cycling treatment on hemiplegic patients: preliminary results. In: Proceedings of the 11th Annual Interna- 
tional FES Society Conference; 2006 Sep 12-15; Miyagi-Zao, Japan. p. 77-9.

35. Ferrante S, Pedrocchi A, Ferrigno G, Molteni F. Cycling induced by functional electrical stimulation improves the muscular strength and the motor control of individuals with post-acute stroke. Europa Medicophysica-SIMFER 2007 Award Winner. Eur J Phys Rehabil Med 2008;44:159-67.

36. Ambrosini E, Ferrante S, Pedrocchi A, Ferrigno G, Molteni F. Cycling induced by electrical stimula- tion improves motor recovery in postacute hemiparetic patients: a randomized controlled trial. Stroke 2011;42:1068-73.

37. Lo HC, Hsu YC, Hsueh YH, Yeh CY. Cycling exercise with functional electrical stimulation improves postural control in stroke patients. Gait Posture 2012;35:506-10.

38. Smart NA, Dieberg G, Giallauria F. Functional electrical stimulation for chronic heart failure: a metaanalysis. Int J Cardiol 2013;167:80-6. 